Documentation et bibliothèques

DOCUMENTATION BIBLIOTHEQUES

\title{
Bibliothèques et archives du Tiers-Monde : problèmes et perspectives
}

\section{Jean de Chantal}

Volume 21, numéro 2, juin 1975

URI : https://id.erudit.org/iderudit/1055500ar

DOI : https://doi.org/10.7202/1055500ar

Aller au sommaire du numéro

\section{Éditeur(s)}

Association pour l'avancement des sciences et des techniques de la documentation (ASTED)

\section{ISSN}

0315-2340 (imprimé)

2291-8949 (numérique)

Découvrir la revue

\section{Citer cet article}

de Chantal, J. (1975). Bibliothèques et archives du Tiers-Monde : problèmes et perspectives. Documentation et bibliothèques, 21(2), 85-95.

https://doi.org/10.7202/1055500ar
Résumé de l'article

L'implantation et le développement des bibliothèques dans le Tiers-Monde connaissent plusieurs problèmes. La formation du personnel professionnel sur place n'est pas réellement possible étant donné le manque d'écoles de bibliothéconomie, ce qui favorise l'émigration de ces spécialistes. Le petit nombre de bibliothèques nationales et leur évolution embryonnaire rend difficile la création de bibliographies nationales qui sont de première nécessité avant que ne disparaisse une partie du patrimoine intellectuel. La nécessité d'organiser de véritables archives ne s'est pas encore fait suffisamment ressentir. En participant à un système international d'information, le Tiers-Monde pourrait ainsi maximiser ses ressources encore insuffisantes.
Tous droits réservés ( $)$ Association pour l'avancement des sciences et des techniques de la documentation (ASTED), 1975
Ce document est protégé par la loi sur le droit d'auteur. L’utilisation des services d'Érudit (y compris la reproduction) est assujettie à sa politique d'utilisation que vous pouvez consulter en ligne.

https://apropos.erudit.org/fr/usagers/politique-dutilisation/ 


\title{
Bibliothèques et archives du Tiers-Monde: problèmes et perspectives*
}

\author{
Jean de Chantal \\ Directeur associé pour le développement des bibliothèques \\ Bureau régional du Centre de recherches pour le développement international \\ Dakar, Sénégal
}

L'implantation et le développement des bibliothèques dans le Tiers-Monde connaissent plusieurs problèmes. La formation du personnel professionnel sur place n'est pas réellement possible étant donné le manque d'écoles de bibliothéconomie, ce qui favorise l'émigration de ces spécialistes. Le petit nombre de bibliothèques nationales et leur évolution embryonnaire rend difficile la création de bibliographies nationales qui sont de première nécessité avant que ne disparaisse une partie du patrimoine intellectuel. La nécessité d'organiser de véritables archives ne s'est pas encore fait suffisamment ressentir. En participant à un système international d'information, le Tiers-Monde pourrait ainsi maximiser ses ressources encore insuffisantes.

There are several problems in the implantation and development of libraries in the underdeveloped countries. The forming of local professionals is not really possible due to the lack of schools of library science, thus favoring the emigration of these specialists. The small number of national libraries and their embryonic evolution make it difficult to create national bibliographies which are of prime need before a part of the intellectual heritage disappears. The need to organize genuine archives has not made itself sufficiently felt. In participating in an international system of information, the underdeveloped countries could thus maximize their resources which are still insufficient.

Existen varios problemas en el tercer mundo con respecto a la implantación y desarrollo de las bibliotecas. No se puede llevar a efecto en esa entitad geográfica la formación de profesionales por falta de escuelas de biblioteconomía, y este fenómeno favorece la emigración de los especialistas en esta materia. El reducido número de bibliotecas nacionales y su evolución embrionaria no facilitan la creación de bibliografias nacionales, que son de primera necesidad antes de que desaparezca una parte del patrimonio intelectual. No se ha experimentado todavia suficientemente la necesidad de organizar un verdadero archivo. Al participar en un sistema international de información, el tercer mundo podria emplear al máximo sus recursos todavia insuficientes.

Tout exposé des problèmes bibliographiques auxquels les pays du Tiers-Monde doivent faire face est nécessairement incomplet tant du point de vue géographique que du sujet lui-même. La tâche, on le comprendra

* N.D.L.R. Adapté d'une conférence prononcée en octobre 1974 devant les élèves du cours Bibliothéconomie comparée de l'École de bibliothéconomie de l'université de Montréal. Les opinions exprimées sont celles de l'auteur et n'engagent pas le Centre de recherches pour le développement international (CRDI). mieux en lisant ces quelques propos, est immense. Aussi, nous limiterons-nous aux quelques pays d'Afrique où nous avons eu le bonheur de travailler quelques années. Les leçons apprises et les solutions envisagées peuvent s'appliquer sans trop de modifications aux problèmes identiques qui entravent le développement des bibliothèques en Asie comme aux Antilles et en Amérique latine.

La situation bibliographique dans certains pays du monde en voie de développement, ou en émergence, ne nous paraît pas 
trop sombre. Bien sûr, il s'y trouve des ombres ici et là: il en faut pour mieux faire ressortir les lumières.

La Conférence internationale sur la bibliographie africaine, tenue à Nairobi en décembre 1967, a adopté pas moins de vingt-trois recommandations. Nous nous attarderons à évoquer seulement quatre des plus grands problèmes de l'heure dans le domaine. L'ordre dans lequel nous les présenterons reflète, à notre avis, la priorité et l'importance que nous leur accordons. II s'agira donc premièrement de la formation bibliographique, deuxièmement des bibliothèques de lecture publique, des bibliothèques nationales et des bibliographies nationales, troisièmement des archives nationales et, finalement, des systèmes internationaux d'information.

\section{La formation bibliographique}

Dans les débuts de l'accession à l'indépendance et surtout avant, l'administration des quelques bibliothèques existantes était confiée aux métropolitains colonisateurs; certains sont encore en poste, heureusement leur nombre décroît. Ce n'est que beaucoup plus tard qu'on s'intéressa sérieusement à la formation des bibliothécaires autochtones. Inévitablement, on rencontra tout de suite de nombreux problèmes: des salaires trop bas n'attiraient que peu de candidats valables à la profession; le bibliothécaire, même celui qui avait la chance d'avoir reçu sa formation professionnelle à l'étranger, se retrouvait très bas dans l'échelle sociale, sans statut reconnu ou défini par le reste de la communauté et, partant, très peu de chances de promotion. Un fonctionnaire influent d'un pays en voie de développement, que nous essayions d'intéresser à l'idée de lancer un programme de formation adéquate pour les bibliothécaires de son pays, disait: «Pourquoi une formation spéciale et professionnelle? Tout ce qu'il vous faut c'est un pion avec un pot de colle et des timbres-dateur en caoutchouc. II n'en faut pas plus pour faire un bibliothécaire!» Résultat: carrière peu attrayante, très petit nombre de bibliothèques par rapport aux besoins pressants, donc pas de demande pour des professionnels, d'où un manque impressionnant d'écoles de formation professionnelle. On voit déjà le terrible cercle vicieux dans lequel on pataugeait.

Pourtant, et presque par miracle, on parvint quand même à s'en sortir. On s'interrogeait, tant du côté francophone qu'anglophone, sur l'opportunité de fonder des écoles locales de bibliothécaires ou de continuer d'envoyer ses étudiants à l'étranger. Cette formation à l'étranger devait tenir compte de certains points de vue, souvent divergents: pourrait-on jamais parvenir à les réconcilier? II y avait le point de vue du gouvernement local qui paie pour cette formation, celui du futur bibliothécaire qui doit partir, celui de la bibliothèque où il travaillera à son retour et celui, enfin, de l'école de bibliothécaires à l'étranger qui sera chargée de sa formation.

Le gouvernement qui subventionne les études à l'étranger d'un de ses ressortissants est très intéressé à ce que ce dernier retourne au pays à la fin de ses études, afin de réduire le plus tôt possible une dépendance coûteuse vis-à-vis des "experts» étrangers ou des programmes de coopération technique. Dans bien des cas, c'est encore le contribuable, le petit payeur d'impôts qui paie pour tout cela. Le gouvernement doit donc s'assurer qu'il n'y a pas de gaspillage, qu'il fait un bon placement, qu'il investit pour l'avenir.

Le futur bibliothécaire voudra, à son retour, entrer dans une carrière professionnelle reconnue, acceptée et convenablement rémunérée.

La bibliothèque qui l'accueillera s'attendra à ce qu'il fournisse des services qui répondent aux besoins de sa clientèle et surtout, qui sont appropriés aux circonstances locales, bien différentes de celles du milieu où il a été formé.

Quant à l'école de bibliothécaires, ses cours sont appropriés aux besoins de la profession tels qu'on les rencontre en pays développé. C'est son rôle précis et l'on ne peut s'attendre à ce que des normes élevées, souvent atteintes au prix de multiples efforts, soient sacrifiées, amenuisées pour accommoder un petit nombre d'étrangers venant de pays en voie de développement.

Cette formation à l'étranger est remplie de pièges. Les solutions préconisées pour un milieu tel que le Canada, par exemple, ne trouvent souvent pas d'application dans une situation de pays en voie de développement. Voici trois exemples parmi tant d'autres.

Les écoles enseignent la classification décimale de Dewey, la Classification décimale universelle (C.D.U.) et celle de la Bibliothèque du Congrès. C'est très bien, c'est l'essentiel. Mais se soucie-t-on assez du peu de flexibilité qui existe dans ces grands systèmes, flexibilité pourtant requise et nécessaire pour classifier les livres qui sont publiés le plus souvent dans le Tiers-Monde et qu'on peut regrouper, gros- 
so modo, sous les quatre rubriques suivantes: philosophie, religion, littérature et histoire?

Nous y prônons l'usage des fiches imprimées par la Bibliothèque du Congrès oubliant que le titre et plus souvent l'édition du volume sur les rayons d'une bibliothèque ghanéenne ne correspondent pas toujours aux données qui apparaissent sur la fiche de Washington.

Les vedettes-matière dont nous recommandons l'usage n'oublient-elles pas trop souvent qu'une langue, ce n'est pas seulement des mots, mais aussi des concepts, des interprétations, des usages qui changent radicalement d'un continent à l'autre, surtout quand ces territoires sont en pleine effervescence et se cherchent encore eux-mêmes?

L'étudiant étranger qui arrive en France, en Angleterre ou qui parvient jusqu'en Amérique du Nord, doit faire face à un climat très différent du sien - au Canada extrêmement dur pour ceux qui viennent des pays tropicaux doit accepter une nourriture étrangère, doit s'assujettir à des méthodes d'enseignement auxquelles il n'a pas été habitué jusqu'alors et qui exigent une bonne dose d'adaptation. Nous connaissons un étudiant africain qui n'a réussi à s'acclimater qu'à la toute veille des examens, alors qu'il devait s'apprêter à rentrer!

Le jeune candidat bibliothécaire, non encore mûri par l'expérience du travail dans son propre pays, risque de ne pas toujours distinguer dans ce qui lui est présenté, non pas entre le bien et le mal, mais entre ce qui est bon et ce qui est moins bon pour son propre pays. Déraciné comme il l'est de son milieu culturel pendant un an, deux ans, il rentre souvent au pays natal la tête gonflée d'idées et d'espoirs pas du tout adaptés et appropriés aux besoins et surtout à la capacité d'absorption de son pays. Un collègue ghanéen est rentré du Canada avec, semble-t-il, une seule idée en tête: se défaire le plus tôt possible du bon vieux système Browne au prêt pour le remplacer par l'appareil à microfilmer en attendant de passer le plus tôt possible à l'ordinateur!

II s'attend en outre, et avec raison, à être reconnu dans son propre pays et exige un statut professionnel qu'on n'est pas encore prêt à lui accorder. Habitué à la "quincaillerie» nord-américaine, tels l'ordinateur, la mécanisation des tâches, la microcopie, la photocopieuse à sous sur tous les étages, il s'étonne de n'en point trouver chez lui. II s'attend à une réaction positive de la part de sa clientèle aux idées nouvelles qu'il apporte, aux méthodes de travail moins onéreuses; il cherche un peu d'appui de la société où il sera appelé à œuvrer, oubliant qu'il n'en tient qu'à lui de créer le climat propice à la transplantation et à l'éclosion de toutes ces idées exotiques importées de l'étranger.

Oublie-t-il que le milieu qu'il retrouve est exactement le même que celui qu'il laissait un ou deux ans auparavant? C'est lui qui a changé: il a été non seulement formé à une nouvelle discipline, mais a été, par surcroît, transformé par le fait même. Qu'il ne s'étonne donc pas si ce milieu n'est pas trop réceptif aux idées nouvelles qu'il rapportera avec lui, qu'il importera en quelque sorte. Pourtant, dès soin retour, il aura comme tâche première d'être un «animateur du changement».

Une telle situation, bourrée de frustrations, chargée de conséquences funestes, conduit souvent le bibliothécaire qui retourne dans son pays, sinon à repartir tout de suite, du moins à se laisser tenter par les offres alléchantes de postes mieux rémunérés, plus reconnus dans une profession autre que celle qu'il avait pourtant choisie et pour laquelle il avait reçu une formation spécialisée. Au moment d'écrire ces lignes, le 16 octobre précisément, le jour de notre arrivée au Sénégal, Le Soleil de Dakar annonçait que le Conseil de Cabinet avait adopté la mesure individuelle suivante: Théodore N'Diaye, professeur certifié, précédemment Directeur des études à l'École des bibliothécaires de l'Université de Dakar, était nommé directeur de la formation permanente au ministère de l'Enseignement supérieur... Une bourse d'études à l'étranger - et ceci ne s'applique pas, hélas, seulement aux bibliothécaires - devient souvent le premier pas vers l'émigration.

Inévitablement donc, devaient s'établir en Afrique et ailleurs dans le Tiers-Monde, au cours des dix dernières années, des écoles de bibliothécaires qui ont fait un progrès remarquable et qui commencent déjà à pallier le manque de main-d'œuvre spécialisée. Elles ne sont pas nombreuses. En Afrique, la plus ancienne est celle de l'Université d'Ibadan dont la fondation remonte à 1958 et qui offre même le doctorat en bibliothéconomie; le $\mathrm{Ni}$ geria en compte aussi une deuxième inaugurée dix ans plus tard à l'Université d'Ahmadu Bello. Le Ghana possède la sienne depuis 1961. Voilà pour l'ouest africain anglophone. Une seule école de bibliothécaires essaie de pourvoir aux beoins de toute l'Afrique francophone: c'est l'École des bibliothécaires, archivistes et documentalistes (E.B.A.D.) de 
Dakar dont l'origine, avec l'assistance de l'Unesco, remonte au début des années soixante.

En Afrique orientale, on retrouve aussi trois écoles: en Éthiopie, en Ouganda où elle dessert l'ancienne communauté est-africaine de l'Ouganda-Tanzanie-Kenya, puis celle de la Zambie. Fait digne de mention, toutes ces écoles, sans exception, sont rattachées à des universités. Comme partout dans le TiersMonde, la situation que nous venons de décrire est en "perpétuelle mutation» pour employer l'expression de Montaigne. Par exemple, certains milieux de Tanzanie et du Kenya expriment déjà des réserves sur l'opportunité d'envoyer leurs bibliothécaires, dont un certain nombre sont d'origine asiatique, se former à l'Université Makerere, au pays du général Amin. La Fondation Ford s'intéresse à un projet qui vise à lancer, en Afrique orientale, un programme d'études supérieures en bibliothéconomie. Un rapport de l'Unesco préconise l'ouverture d'une autre école de formation francophone; celle-là au Congo. Sur l'autre rive du fleuve Congo, selon le directeur de l'E.B.A.D., I'Université nationale du Zaïre envisage de lancer, dans un proche avenir, une section de formation de bibliothécaires dans le cadre de l'Institut des techniques de l'information qui forme présentement surtout des journalistes: intéressante conjoncture de deux disciplines de la science dite de l'information. A l'Université de l'lle Maurice, on instaurera incessamment un cours expérimental de formation d'assistants-bibliothécaires qui empruntera certains éléments aux cours dispensés aux bibliotechniciens nord-américains, mais avec ceci de particulier qu'on essaiera de former en même temps des animateurs qui iront s'intégrer à la vie de village dans le cadre d'un important projet de développement rural, financé par la Banque mondiale. Le Centre de recherches pour le développement international s'est intéressé à cette initiative et contribuera financièrement à son lancement.

En terminant ce chapitre sur la formation, nous voudrions évoquer quelques-uns des problèmes qui ont retenu l'attention des directeurs de la plupart des écoles africaines de bibliothécaires, réunis pour la première fois à Dakar en février 1974 grâce à l'initiative du directeur de l'E.B.A.D. Il y a d'abord la difficulté de recruter des professeurs africains de bibliothéconomie; les meilleurs praticiens ne deviennent pas nécessairement de bons professeurs: c'est un lieu commun de le répéter. Les participants à ce séminaire profitèrent donc de l'occasion pour demander une aide à l'Unesco afin de hâter la formation de professeurs africains de bibliothéconomie. On évoqua ensuite les problèmes posés par l'enseignement de matières très particulières au contexte africain, pour lesquelles n'existe encore aucun manuel approprié: la catalographie des auteurs africains aux patronymes très souvent complexes, l'histoire et le développement des bibliothèques en Afrique. L'isolement des écoles, rendu plus grave, tant par les distances géographiques que par la barrière des langues, fit aussi l'objet d'une étude particulière qui recommanda l'adoption de mesures favorisant l'échange des professeurs tout comme celui des étudiants, entre pays africains au départ et, plus tard, pourquoi pas, à l'échelle intercontinentale.

\section{Bibliothèques et bibliographies nationales}

Comme on le verra plus loin au chapitre des archives, les bibliothèques nationales, là où elles existent dans les pays en voie de développement, évoluent parfois dans un décor extrêmement confus. Depuis de nombreuses années, les missionnaires et leurs maisons d'édition impriment localement un fort volume de publications assez spécialisées. À cela, viennent s'ajouter les organisations volontaires: fermiers, syndicats, associations féminines, etc. Cependant, beaucoup d'écrits touchant l'Afrique sont encore publiés à l'étranger, même des œuvres d'auteurs africains eux-mêmes, phénomène d'ailleurs qui n'est pas étranger au Québec. Les maisons d'édition africaines sont encore assez humbles, pour la plupart; quelques grandes firmes des anciennes métropoles essaient bien d'y implanter des succursales; leur succès, même dans le cas où la gérance est confiée à un personnel africain, nous semble aléatoire. Les librairies africaines trouvent plus de profit à vendre des volumes importés qu'à afficher des publications nationales dans leurs vitrines. La nature et la fonction des bibliothèques nationales doivent donc être examinées en tenant compte de cette situation assez complexe.

Point n'est besoin, pour une bibliothèque nationale, d'en avoir le nom pour en jouer le rôle. Bien sûr, il y a la "Nationale» de Paris, mais il y a aussi le British Museum, la Bibliothèque du Congrès, la Bibliothèque Royale Albert 1er, la Deutsche Bibliothek, la Bibliothèque de l'Institut à l'lle Maurice et combien d'autres encore. II s'en trouve qui répondent, sous le même toit, aux besoins des archives nationales, de l'unique université du pays tout en réservant une place importante à la lecture 
publique. Comme partout ailleurs, elles essaient de rassembler tout ce qui est publié non seulement dans le pays même, mais en plus les œuvres sur le pays et qui s'écrivent à l'étranger, que ce soit par des nationaux émigrés ou par d'autres. Cette première tâche devient assez facile quand le dépôt légal existe et qu'il est mis en vigueur; quant à l'autre, ce n'est pas facile. II existe des endroits où la "Nationale» est la seule bibliothèque digne de ce nom sur tout le territoire. Par ailleurs, on trouve aussi des pays aux nombreuses bibliothèques où la "Nationale" est la plus démunie de toutes, tant en ressources humaines que financières. Il y a un rayon d'espoir, cependant: ce sont les bibliothèques nationales qui entreprennent, d'habitude, la publication de la bibliographie nationale et qui, la plupart du temps, réussissent le tour de force de sortir un travail qui se compare favorablement, toutes proportions gardées, aux grandes œuvres issues des grands pays aux gros ordinateurs.

Prenons le cas de la Guyane anciennement britannique. La Bibliothèque nationale est issue de la bibliothèque centrale de lecture publique, fondée à Georgetown par Andrew Carnegie. La bibliographie nationale, dont la publication ne remonte qu'à 1973 seulement, renferme absolument tous les éléments essentiels: description du contenu, référence à la loi du dépôt légal, explication de la classification employée, liste des abréviations, liste des maisons d'édition guyanaises avec leurs adresses et numéros de téléphone. La description bibliographique extrêmement complète est rédigée selon les règles de l'Anglo-American Cataloging Rules. A l'autre extrémité du monde, l'on n'a qu'à consulter la bibliographie nationale de la Côte d'Ivoire pour constater ce qui peut se faire avec des effectifs humains et financiers beaucoup plus considérables.

La tendance actuelle dans le monde est, pour chaque pays, d'avoir sa propre bibliothèque nationale; il y a eu des retardataires et pas seulement dans le Tiers-Monde: le Canada n'aménagea dans la sienne, c'est-à-dire dans des locaux adéquats, qu'en 1967. En principe, certains pays moins nantis, face à la pénurie de personnel qualifié et aux minces ressources financières disponibles, pourraient mettre leur avoir en commun; en pratique cependant, l'orgueil national rendrait tout espoir de collaboration de ce genre quasi irréalisable.

La tendance, en Afrique surtout, semble d'établir une multiplicité de bibliothèques dans un milieu qui pourrait plus efficacement être desservi par une seule. Même si, de prime abord, l'idée pourrait paraître saugrenue, pourquoi ne serait-il pas possible d'imaginer une construction à usages multiples à quatre portes ainsi marquées: Bibliothèque nationale, Bibliothèque publique, Bibliothèque universitaires, Archives nationales?

Comme c'est le cas pour les archives, on le verra plus loin, la mise à la disposition du public d'une lecture saine par l'entremise de bibliothèques bien gérées ne peut que contribuer au développement socio-économique d'un pays. Une fois les politiciens convaincus de ce principe fondamental, ils verront bien à trouver les fonds nécessaires à l'établissement de telles bibliothèques et à la formation tout aussi importante de leur personnel. L'approvisionnement en livres et en ouvrages de référence sera ensuite assuré lors de l'établissement des prévisions budgétaires des plans de développement économique. L'un de ces gros volumes que nous consultions récemment, justement pour vérifier jusqu'à que point on accordait de l'importance au rôle que peuvent jouer les bibliothèques dans ce domaine, ne mentionnait le mot "bibliothèque» qu'une seule fois en quelque neuf cents pages!

Ouvrons ici une courte parenthèse consacrée aux bibliothèques de lecture publique. II existe, entre les nombreuses régions du monde en voie de développement, des différences dramatiques du point de vue des bibliothèques et du genre de services qu'elles dispensent. On peut trouver, dans les centres métropolitains et dans les établissements d'enseignement supérieur, des bibliothèques qui reflètent la longue tradition de savoir de l'Asie, de l'Amérique Latine et du monde islamique. À quelques exceptions près, les bibliothèques en Afrique noire sont, de loin, les moins bien développées. Faut-il ajouter que partout dans les pays en voie de développement l'accès aux bibliothèques est souvent soumis à des conditions restrictives, probablement nécessaires en raison du coût des livres. Dans plusieurs de ces mêmes pays, le conservateur de la bibliothèque est encore financièrement responsable de tous les livres qui se trouvent dans sa bibliothèque et il lui incombe de payer de sa poche les livres achetés en remplacement de ceux qui sont perdus ou volés; d'où l'on voit toute la force que prend le mot "conservateur», véritable gardien de la bibliothèque.

Cette situation va, d'une façon notoire, à l'encontre de la satisfaction de ce désir toujours croissant qui pousse les gens vers la lecture, désir particulièrement propre aux jeu- 
nes soucieux d'acquérir des connaissances dont ils peuvent se servir pour vaincre la pauvreté et l'insécurité, afin de contribuer ainsi à leur propre émancipation.

De plus en plus, les gens considèrent la bibliothèque, plus particulièrement les bibliothèques publiques, comme un facteur positif d'éducation, d'innovation et même d'action sociale et non pas seulement comme un magasin où sont entassés des livres. Les bibliothécaires, surtout ceux des pays en voie de développement, doivent être toujours prêts à donner satisfaction à l'usager qui demande des renseignements. Naturellement, ceci peut être accompli par le prêt d'un livre, mais pour les groupes sociaux qui, normalement, comptent sur une communication orale, le bibliothécaire doit être prêt à trouver d'autres moyens d'action. II peut être l'allié de l'instituteur qui combat l'analphabétisme, l'ami du travailleur social qui fournit des renseignements sur l'hygiène ou le planning familial, le collaborateur du spécialiste qui œuvre dans le domaine de la formation professionnelle.

II nous semble qu'un plus grand nombre de livres dans les langues vernaculaires pourraient trouver place sur les rayons des bibliothèques de ces pays, quitte à déplacer pour cela des romans occidentaux qui peuvent n'apporter aucun enrichissement à ces populations. Nous nous rappelons avoir vu à la bibliothèque publique d'Accra, section des jeunes, le Christmas Carol de Charles Dickens où un abonné des plus patients a souligné tous les mots nouveaux rencontrés et en a inscrit très proprement la définition dans la marge, pour l'édification des lecteurs à venir. C'est ainsi qu'au mot "snow" on pouvait lire "eau congelée dans les hautes régions de l'atmosphère et qui tombe en flocons blancs et légers»: de quoi faire rêver tous ces petits Africains d'un Noël tout blanc!

II y aurait lieu d'accorder aussi beaucoup plus d'importance aux livres du genre dit «Faites-le vous-même»: comment construire sa propre case, par exemple, à l'aide de matériaux bon marché et trouvés sur place.

Nous affirmions donc, avant d'ouvrir cette parenthèse sur la lecture publique, que les bibliothèques nationales ou, à défaut, celles qui remplissent ce rôle dans les pays en voie de développement, publient d'ordinaire la bibliographie nationale du pays, l'inventaire du patrimoine intellectuel de la nation. Au Canada, c'est Canadiana, en Angleterre, la British National Bibliography, en France, la Bibliographie de la France, en Côte d'Ivoire, la Bibliographie de la Côte d'lvoire, etc. Nombreux sont les pays qui ne se sont pas encore souciés de ce devoir, ou qui n'en n'ont pas les moyens et qui sont néanmoins conscients du danger de laisser échapper ainsi, de manière irrémédiable peut-être, une documentation de portée historique et culturelle de valeur inestimable. Certains pays des Antilles du Commonwealth, préoccupés par cette question, ont décidé de regrouper leurs efforts, car une solution fragmentée du problème serait évidemment malencontreuse.

Chacun de ces territoires - ils sont quatre, en l'occurrence - est intéressé non seulement par sa propre littérature mais aussi par celle des territoires avoisinants puisque, malgré leur disparité, leur inégalité et leur éloignement, ils font quand même tous partie d'une même région et d'une même famille de nations: les Antilles occidentales du Commonwealth britannique. II s'agit de la Guyane qui, comme on l'a vu plus haut, possède déjà sa propre bibliothèque nationale, de la Jamaïque qui est à la veille d'avoir la sienne, de la Trinité-Tobago et de la Barbade qui ne devraient pas tarder à emboîter le pas.

Admettons que chacun de ces petits pays tombe d'accord pour établir sa propre bibliographie nationale et accepte de se conformer aux mêmes normes de rédaction: quelle magnifique perspective s'ouvre alors à nos yeux de voir quatre bibliographies nationales pouvoir s'intégrer parfaitement et sans heurt en une bibliographie régionale capable ensuite d'accueillir à son tour les contributions d'autres territoires prêts à accepter des normes qui ont déjà fait leurs preuves.

L'Unesco, et plus précisément la FIAB à Grenoble en 1973, ont toutes deux mis de l'avant le fameux concept du CBU (contrôle bibliographique universel) et en ont réglé les dispositions: I'ISBD (description bibliographique internationale normalisée), l'ISSN, I'ISBN, etc. deviendront rapidement des signes connus de tous. Le CRDI, ayant été saisi d'une requête officielle de ces pays, accorda donc son appui à une première rencontre qui eut lieu à la Jamaïque à la fin du mois d'avril 1974, rencontre convoquée pour examiner de plus près les modalités d'une telle expérience. Tous les participants qui étaient, d'une part les quatre rédacteurs de la bibliographie nationale de chacun des territoires, d'autre part un représentant de chaque gouvernement en prévision du temps où il faudra aller chercher des fonds pour la mise en œuvre des projets - sont tombés d'accord pour tenter l'expérience pendant les prochains six mois et pour se réunir une autre fois en novembre 1974 afin de procéder à une évaluation des 
résultats et aplanir les difficultés rencontrées pour mieux démarrer dès janvier 1975. Cette réunion de novembre, à la différence de la première, groupa les catalogueurs et les responsables de la publication bibliographique: ceux qui mettent la main à la pâte, en quelque sorte. On prévoit, en outre, la présence d'un délégué de la FIAB et d'un catalogueur travaillant déjà à la préparation d'une bibliographie nationale mettant en application les règles de I'ISBD. Ces deux experts devraient avoir toutes les réponses aux problèmes rencontrés au cours de la phase expérimentale. Si l'essai antillais s'avère un succès et si d'autres régions du Tiers-Monde désirent se prévaloir des leçons apprises, il sera alors facile d'adapter et d'adopter la formule ailleurs.

Après cela, l'emploi des ordinateurs pour la mise en mémoire et l'exploitation des données bibliographiques, l'échange de ces données sous forme lisible en machine ou sur fiches perforées ainsi que leur consultation à distance, à l'échelle internationale grâce aux satellites qui sont déjà en place, ne seront qu'un jeu. Le jour n'est pas loin - n'y sommes-nous pas déjà avec le "Cataloguing in Process" de la Bibliothèque du Congrès? où chaque publication imprimée ou graphique, sonore ou électronique sera cataloguée une fois pour toutes dans son pays d'origine et paraîtra dans la bibliographie nationale de cet état. Les centres bibliographiques qui en ont les moyens - ce n'est pas, évidemment, le cas de nombreux pays en voie de développement - traiteront l'information en machine pour la redistribuer sur leur propre territoire et à l'étranger, soit sous forme de bandes perforées ou magnétiques ou sous toute autre forme de mémoire transmissible que les progrès techniques imposeront, soit sous forme de fiches imprimées. Les pays moins nantis n'auront qu'à faire comme à présent: distribuer des exemplaires de leurs bibliographies nationales aux autres, avec l'entente préalable qu'un pays plus fortuné dans une région se charge de la mise en mémoire de la production bibliographique de ses voisins. La coopération internationale et la normalisation des règles éviteront toute nouvelle rédaction des notices bibliographiques et toute distribution unilatérale de notices préparées à partir d'un choix, même s'il est quasi universel (est-ce alors un choix?) comme celui de la Bibliothèque du Congrès. La coopération internationale doit être établie, dès le départ, sur un pied d'égalité.

L'emploi généralisé des ordinateurs imposera nécessairement une adaptation des règles actuelles de catalogage à leur entrée en machine; heureusement, il ne sera pas question de remettre en cause les principes fondamentaux du catalogage auteurs ou matières. Cet emploi de la machine suggère donc des modifications aux usages les plus profonds. Aucun des codes traditionnels de catalogage n'a été pensé en vue de l'automatisation; il faudra donc repenser et préciser les règles actuelles. D'autre part, de grands projets comme UNISIST de l'Unesco n'accordent pas au nom de l'auteur l'importance qu'on lui donne traditionnellement dans les catalogues de bibliothèques et proposent des notices commençant par le titre. II faut être au courant de ces projets pour en suivre le développement.

Nous ne nous attarderons pas ici sur l'importance, pour tous les pays, d'accepter la numérotation internationale du livre (ISBN) le plus rapidement possible. Ce chiffre fournit un moyen commode et sûr d'identifier les différents titres et les différentes éditions du même titre. Il faudra donc accélérer son adoption par les groupements d'éditeurs de chaque pays et par les bibliographies nationales aussi bien dans les "vient de paraître» que dans les notices bibliographiques. Un jeune auteur d'un pays africain, observateur perspicace avouons-le, nous offrit un jour un exemplaire de sa dernière œuvre. Tout en le félicitant de la bonne présentation de son livre, nous remarquâmes au dos de la page de titre un étrange assemblage de lettres et de chiffres précédés de la mention ISBN. Sachant que le système de numérotation internationale n'était pas encore en vigueur chez lui à ce moment-là, nous lui demandâmes comment il était parvenu à composer "son" chiffre. II nous répondit tout bonnement qu'il avait remarqué que dans tous les récents livres publiés en Angleterre et aux États-Unis, se trouvaient ces chiffres alignés au dos de la page de titre; s'étant renseigné, il apprit que, dorénavant, ce numéro aurait une importance considérable dans la mécanisation des commandes, qu'il ferait partie désormais de tous les catalogues d'éditeurs, etc. II crut donc bien faire en y allant du sien, puisant les éléments de son numéro dans sa date de naissance en ayant soin d'y joindre ses initiales!

Toutes ces modifications laissent supposer un changement de mentalité chez de très nombreux bibliothécaires et une révision profonde de leurs habitudes de travail. Ces changements sont, de par leur nature, généralement lents. Un travail de préparation psychologique devrait être entrepris, particulièrement auprès des jeunes bibliothécaires pendant leur formation professionnelle. Chez les 
moins jeunes, cela pourrait prendre encore plus de temps...

Pour conclure cette partie, nous emprunterons à John Dean, ancien bibliothécaire à l'Université du Ghana et fondateur de l'École de bibliothécaires d'Ibadan, les raisons principales qui, selon lui, retardent le développement des bibliothèques dans ces pays du Tiers-Monde. Ces facteurs que l'on pourrait appeler d'ordre extérieur, dus à la nature, à des conditions socio-économiques, ou au passage de la colonisation, sont les suivants: isolation résultant des mauvaises communications, climat difficile, difficultés politiques, économiques et financières qui se reflètent dans des budgets réduits, absence de toute habitude de lecture, de fréquentation des bibliothèques à l'école, à la maison, au travail, absence totale, dans bien des cas, d'une infrastructure solide au niveau de la bibliothèque nationale et des bibliothèques de lecture publique, manque de spécialistes en matière de réparation et de mise en marche des équipements techniques qui deviennent indispensables, de nos jours, dans toute bibliothèque qui se veut moderne. Ajoutons à cela ces quatre facteurs d'ordre «intérieur" et l'on comprendra mieux la tâche de ceux qu'intéresse le progrès des bibliothèques en pays en voie de développement: pénurie de fonds suffisants, manque de personnel qualifié, négligence dans la planification du développement à long terme, prédominance des idées périmées en matière de bibliothèque et de documentation.

\section{Archives}

Les archivistes des pays en voie de développement, tout comme les bibliothécaires, d'ailleurs, ont à faire face à deux problèmes majeurs avant de parvenir au contrôle bibliographique de leurs manuscrits et de leurs documents: premièrement - cela paraîtra simpliste - le simple ramassage et la mise en ordre des pièces déjà sur place, mais souvent éparpillées sur toute l'étendue du territoire; deuxièmement, le rassemblement des originaux, ou au moins des copies, des archives et des annales dispersées aux quatre coins du monde. II n'existe probablement aucune autre civilisation sur terre dont le passé écrit soit autant disséminé que celui de l'Afrique, à un point tel que l'histoire de l'Afrique ne peut s'écrire à moins d'aller consulter en Arabie les relations des premiers explorateurs, les archives de France, d'Angleterre, d'Allemagne, du Portugal, d'Espagne et même des États-Unis. Quelles perspectives pour l'usage plus généralisé des microcopies!
Les archives bien organisées peuvent effectivement contribuer de plusieurs façons au développement économique et social d'un pays. Le directeur des Archives publiques du Canada, après avoir évoqué, dans un article publié il y a quelques années, pas moins de dix raisons qui font que les archives nationales des pays en voie de développement sont souvent, et par nécessité dans bien des cas, reléguées au bas de l'échelle des priorités, nous propose quatre liens très étroits qui peuvent être établis entre un service d'archives bien organisé et l'efficacité administrative d'un gouvernement, le développement économique d'un pays et son unité nationale.

En premier lieu se trouve le fameux «records management" tellement en vogue en Amérique du Nord, la "gestion des dossiers", comme on pourrait l'appeler pour ne pas s'enliser dans l'anglais. Combien d'heures, de jours, de semaines parfois ne doit-on pas attendre qu'on fouille dans des dossiers en désordre avant de trouver un document? Un système de dossiers bien organisés permettrait, en outre, un programme de destruction rationnelle des papiers sans valeur et la conservation en microcopie ou en archives des documents importants. La vie trop brève des documents éphémères, surtout en Afrique, est imputable, la plupart du temps, davantage aux circonstances de leur publication et de leur diffusion qu'à un manque d'intérêt. Dans beaucoup de pays, l'édition en tant qu'activité industrielle est encore au stade embryonnaire, et des publications de toutes sortes sont ou multigraphiées ou imprimées en petits tirages sur des presses locales. Elles s'épuisent rapidement et elles deviennent alors introuvables avant même d'être signalées dans les bibliographies. Voilà un autre défi lancé à ceux qui se destinent au travail de bibliothécaire dans ces régions.

Comment, en deuxième lieu, les archives peuvent-elles contribuer au développement économique et social d'un pays? II est manifestement clair qu'il faut bâtir sur l'expérience du passé, fût-elle courte, en éviter les erreurs et écarter le double emploi des minces ressources humaines et matérielles. L'auteur de l'article cité plus haut cite l'exemple de plans et devis ayant trait à la construction d'une route au Ghana, dont les travaux durent être interrompus pendant quelques années. Entretemps, les documents furent égarés et il en coûta un demi-million de dollars pour recommencer les plans. De nombreux rapports détaillés sur la culture du riz et du ver à soie, qui ont pourtant considérablement contribué au développement de ces deux industries à 
Madagascar, sont restés longtemps cachés, inaccessibles à d'autres pays qui auraient pu en bénéficier. Les vieilles cartes géologiques révèlent souvent l'emplacement de gisements importants non exploités faute de routes, de marchés ou d'équipements suffisamment perfectionnés; aujourd'hui, face à la pénurie des matières premières, ces dépôts, à condition de retrouver les vieilles cartes, pourraient devenir rentables grâce à la technologie moderne et aux besoins toujours croissants des marchés mondiaux. Ailleurs, de vieux registres de la police ont permis d'établir l'harmonie ou son absence - qui pouvait exister entre différentes ethnies dans un territoire où cherchait à s'implanter une grande industrie en quête de main-d'œuvre où l'entente devait être de rigueur. On peut donc affirmer, sans trop se tromper, qu'un service d'archives bien organisé facilite le planning économique, réduit les pertes occasionnées par le double emploi et les erreurs antérieures, assure la continuité et l'uniformité dans les méthodes, place enfin à la disposition des chercheurs, des économistes, des savants, toute l'expérience du passé dans un cadre bien défini.

Les archives peuvent-elles finalement resserrer les liens de l'unité nationale? On sait que bon nombre d'états de création récente ont été fondés par des puissances coloniales pas toujours soucieuses, dans le découpage - pour ne pas dire le dépeçage - des continents d'Afrique, d'Asie et des Amériques, de la répartition des régions ethniques et bien souvent des frontières naturelles. Le vacuum laissé par le retrait - seulement apparent dans bien des cas - des anciennes métropoles, a encouragé des rivalités profondes entre les peuples, les tribus et les individus eux-mêmes; des luttes ancestrales furent ranimées; des éléments étrangers sont intervenus sournoisement dans bien des cas pour compromettre sérieusement l'avenir d'un jeune pays. D'où ce besoin intense de nouer, le plus tôt possible, des liens d'unité et d'identité nationale. Comment? En puisant dans des archives bien gérées, des documents et des éléments qui serviront à écrire l'histoire du pays.

De bonnes archives peuvent être, en outre, d'une valeur inestimable pour la promotion de l'éducation dans un Tiers-Monde qui est pressé de rattraper le temps perdu. Dans bien des domaines, il devra encore se contenter de faire venir du prêt-à-porter de l'étranger; pas pour son histoire, cependant. Les manuels africains d'histoire puisent de plus en plus dans les archives locales; on apprend la géographie avec l'audio-visuel qui s'inspire des sources de l'histoire au pays. Et ce processus dépasse le cadre de l'école, du collège, de l'université; ce faisant, il devient un instrument d'unité et d'identité nationale. Expositions, conférences, diapositives, films, radio, télévision: tous les moyens sont dorénavant utilisés. On s'attache à recueillir la tradition orale avant qu'elle ne disparaisse complètement: folklore, musique, danse. Un sage du Mali prophétisait: "Dans l'Afrique d'aujourd'hui, chaque vieillard qui meurt, c'est une bibliothèque qui brûle». La sentence vaut aussi pour d'autres régions du monde.

Si donc l'on reconnaît et, fait plus important, si l'on réussit à faire apprécier le rôle primordial que peuvent jouer les archives dans le développement économique d'un pays, quelle procédure reste-t-il à suivre pour mettre en œuvre un tel système? Elle se résume à trois principales étapes: si l'on ne trouve pas sur place les spécialistes pour diriger les travaux et convaincre les autorités, il faudra alors faire appel à l'assistance technique étrangère. II faudra ensuite exiger du gouvernement un engagement formel de trouver les ressources nécessaires à la bonne marche du projet. La troisième condition qui joue en quelque sorte le rôle de clef de voûte, c'est la formation professionnelle et technique pour assurer, dans chaque pays, non seulement le bon fonctionnement des archives, mais aussi une certaine continuité dans l'opération d'un système d'archives adapté aux besoins particuliers de chaque pays, de sorte qu'il devienne institution vraiment nationale et non article d'importation. Pour m'exprimer en termes fleuris, les archives seront, alors, moins une implantation étrangère qu'une greffe salutaire d'un organe enfonçant profondément ses racines dans le sol natal.

\section{Systèmes internationaux d'information}

Les pays en voie de développement n'échappent pas, eux non plus, à cette autre explosion: non pas l'explosion démographique, mais celle de la documentation, véritable marée engloutissante de livres, de périodiques, de journaux et de documents de toutes sortes. Face à cet envahissement, commencent à se dessiner certains efforts à l'échelle internationale qui, s'ils n'apportent pas encore des solutions définitives, en indiquent cependant la voie et commencent à en régler le mécanisme.

Le système d'information idéal est celui auquel on peut poser une question et qui fournira une réponse basée sur les renseignements les plus récents et les plus précis. La mise en place d'un tel système exige un travail considérable et coûteux. Rien ne peut en sortir 
qui n'y ait d'abord été placé. Constituer un fichier de plusieurs centaines de milliers de références coûte plusieurs millions de dollars (dix dollars par référence n'est pas un coût excessif). C'est donc dire qu'une telle entreprise est hors de portée des "fondations" ou des organisations les mieux nanties. D'où la nécessité de partager le fardeau fiscal pour répartir les dépenses; il s'ensuit une décentralisation des tâches, chacun des participants du système ne préparant que les entrées qui décrivent soit les publications de son propre territoire, soit celles qui relèvent d'une compétence particulière.

Le premier de ces grands systèmes est celui de l'information sur l'énergie nucléaire: INIS (International Nuclear Information System) parrainé par l'Agence internationale de l'énergie atomique, dont le siège social est à Vienne. II fonctionne depuis trois ans et s'enrichit de quelque 60,000 éléments d'information chaque année. Ce système étant placé sous l'égide des Nations Unies, chaque participant a voix au chapitre.

Suite aux progrès réalisés par INIS, l'Organisation des Nations Unies pour l'agriculture et l'alimentation (FAO) a commencé à étudier la possibilité de mettre au point un autre système qui traiterait la documentation mondiale relative aux sciences et à la technologie agricoles. II a reçu le nom d'AGRIS et devait démarrer en janvier 1975, à moins d'imprévus. La formule de participation au système AGRIS sera quelque peu différente de celle d'INIS étant donné non seulement la profusion des éléments d'information pouvant atteindre facilement 250,000 par année, mais aussi la nature infiniment plus complexe des organismes à caractère agricole dans l'ensemble du monde. Grâce aux normes internationales déjà acquises, les dispositions techniques d'INIS ont servi à lancer, dès août 1973, une liste expérimentale de démonstration d'AGRIS.

L'Unesco a mis en place un cadre assurant une coopération internationale permanente pour l'élaboration de systèmes efficaces d'information. Ce programme, connu sous le sigle UNISIST, prévoit une série de services communs pouvant apporter un soutien précieux non seulement à INIS et à AGRIS mais à d'autres systèmes à venir. Par exemple, avec la collaboration du gouvernement français, I'UNISIST a installé à Paris un centre qui aura pour tâche de constituer un registre des périodiques scientifiques et techniques du monde entier et qui diffusera pour ceux-ci des indicateurs communs.
Quelle sera la prochaine étape? Dans quelle direction devrions-nous maintenant concentrer nos efforts? Le prochain système international d'information pourrait, par exemple, répondre aux besoins de la vaste communauté internationale que composent tous ceux que préoccupe le développement économique et social, que ce soit au niveau de la planification des programmes, de leur mise en œuvre, de leur évaluation ou de la recherche. Ceux qui ont travaillé au développement connaissent les difficultés d'obtenir des renseignements sur ce qui a déjà été réalisé antérieurement. II existe bien quelque 300 revues consacrées aux informations scientifiques sur le développement et à l'analyse de la situation économique et sociale dans les pays en voie de développement; cependant, une quantité encore plus considérable de renseignements demeure enfouie dans les documents miméographiés rédigés à la suite d'études, de missions et de projets, données qui ne sont répertoriées dans aucun des systèmes existants, d'où gaspillage, répétition, perte de temps et d'énergie. Ce nouveau venu dans la famille internationale des systèmes d'information a nom DEVSIS (système d'information scientifique sur le développement). II tirera profit de l'expérience déjà acquise par INIS et AGRIS et se fondera sur les principes établis par UNISIST.

Cette proposition soulève immédiatement une série de questions d'ordre pratique: quelle sera la formulation détaillée du système? Qui y participera? Quels mécanismes peut-on mettre en place pour la gestion et la coordination du système et l'interclassement des entrées? Comment sera-t-il financé? Quelles seront les limites du sujet? Avec quelle précision seront-elles définies? Pourra-t-on ou devra-ton introduire des renseignements confidentiels dans le système? Si oui, comment serontils protégés? Comment pourra-t-on concilier les divers besoins des dirigeants des pays en voie de développement, des organismes de coopération technique et des agences multinationales? Quels seront les types de sortie les plus utiles? Dans quelle mesure le monde du développement souhaite-t-il vraiment disposer d'un système qui lui permette de retrouver les informations qu'il aura lui-même enregistrées? Dans quelle mesure les gouvernements et organismes donateurs et bénéficiaires ont-ils la volonté de partager leurs renseignements?

Pour trouver une solution à ce problème et pour définir les objectifs de DEVSIS, une conférence internationale réunissait à Ottawa, 
en juin 1974, les représentants de vingt-huit organismes nationaux et internationaux. Ces objectifs sont au nombre de quatre:

a) permettre aux individus comme aux organismes des pays en voie de développement et aux autres intéressés de disposer d'un meilleur moyen d'accès à l'information économique et sociale;

b) favoriser la mobilisation des ressources nationales et internationales qui sont indispensables pour atteindre cet objectif;

c) améliorer la coordination entre les services d'information déjà existants en matière de développement;

d) éviter les répétitions inutiles et le gaspillage des ressources.

Un comité d'organisation et un groupe de travail sont déjà à l'œuvre pour étudier la possibilité d'un tel système. Les augures sont bons et l'avenir est prometteur.

On est donc loin des constatations pessimistes de Lester Asheim qui, en tant que directeur du Bureau des relations internationales de l'American Library Association notait, en 1966, il n'y a même pas dix ans de cela, les lacunes de quelques bibliothèques qu'il avait visitées des pays en voie de développement: "Trop de collections de livres qui $n$ 'ont encore servi à aucun chercheur, trop de marbre extérieur cachant des intérieurs non encore terminés, trop de rayonnage fermé à clef, trop de fichiers qui sont beaucoup plus une énigme qu'un guide pour l'usager, trop de machines qui ne.fonctionnent pas, faute de pièces de rechange introuvables sur place ou de personnel qualifié, trop d'ouvrages de référence tous neufs qui n'ont jamais été consultés, trop de collections de périodiques avec d'importants vides au milieu ou dont les abonnements ont cessé à des dates coïncidant, dans les deux cas, avec l'interruption ou la cessation d'un programme d'assistance technique étrangère».

Les temps ont beaucoup changé. Les problèmes sont en voie d'être réglés. Les perspectives sont bonnes.

\section{Ouvrages consultés}

Asheim, L. Librarianship in the Developing Countries. Urbana, University of llinois Press, 1966.

Chevrier, Jacques. Littérature nègre. Paris, Colin, 1974 (Collection U Prisme). Les deux derniers chapitres traitent de la tradition orale et écrite ainsi que du livre et de la lecture en Afrique.

Dean, John, "Organization and services of university libraries in West Africa" in M.M. Jackson, ed. Comparative and International Librarianship. Westport, Conn., Greenwood Pub. Corp., 1970.

Honoré, Suzanne. Les implications futures de l'automatisation sur le catalogage. Document de travail no 13. International meeting of cataloguing experts, Appendix 3. Copenhague, 1969.

Panofsky, Hans E., «National libraries and bibliographies in Africa" in M.M. Jackson, ed. Comparative and International Librarianship. Westport, Conn., Greenwood Pub. Corp., 1970

Pearson, J.D. and Ruth Jones, eds. The Bibliography of Africa; proceedings and papers of the International Conference on African Bibliography, Nairobi, 4-8 December 1967. London, Frank Cass, 1970.

Smith, Wilfred I. "Archives in developing countries: a contribution to national development». American Archivist, vol. 35 (April 1972).

Woolston, John E. Le DEVSIS: un système d'informations scientifiques sur le développement. Communication préparée à l'occasion d'une réunion de travail tenue à Ottawa en juin 1974. (IDRC-doc-041, janvier 1974).

le CRDI informe, vol. 2, no 1 (mars 1973). (Numéro spécial consacré à la Direction des sciences de l'information) 